\title{
Computational Processes in Iterative Stochastic Control Design
}

\author{
Semoushin I. V. ${ }^{1}$ and Gorokhov O. Yu. ${ }^{1}$ \\ Ulyanovsk State University, L. Tolstoy Str. 42, 432700 Ulyanovsk, Russia
}

\begin{abstract}
In this article we analyse computational processes and investigate characteristics of iterative stochastic algorithms for control and identification based on the auxiliary performance index (API) approach. A workable API is the one which (1) depends on available values only, (2) has the same domain as the original performance index (OPI) has, and (3) achieves its minimum at the same point in the domain as the OPI does. In the article we show that the proposed method for replacing the OPI by an API is applicable for identification the discrete-time steady-state Kalman filter included into the closed-loop stochastic control systems.
\end{abstract}

\section{Introduction}

In order to obtain a good model for control under the real conditions of uncertainty, it has been shown that iterative design procedures are adequate [1]. One iteration involves direct identification of plant model from experimental data and replacement the old model by the new one. Thus the question of identifiability is central in the procedure. This question is twofold: theoretical identifiability and practical identifiability. Basic concepts of theoretical identifiability for plants described by linear difference equations with constant coefficients are set forth in [2]. Numerical methods of parameter identification have also received much consideration, e.g., [3], [4]. A review and unification of linear identifiability concepts can be found in [5].

System identification combines system modelling with parameter estimation. In-depth coverage of system identification in its first unified and mathematically rigorous exposition, including the new general theory of Minimum Prediction Error (MPE) identification methods, is given by Caines [6]. Consistency and other basic qualitative properties of parameter estimators have been thoroughly analyzed, e.g., for a broad class of MPE methods in [6] and [7].

In order to relate the contribution of this paper to the existing control systems literature, we emphasize the fact that in classical MPE methods [6] the set of models $\mathfrak{M}(\theta)$ with a vector parameter $\theta$ is constructed to predict the given, i.e., available observation process $y$, for example, $y=\left[\begin{array}{l}z \\ u\end{array}\right]$ as for closed-loop control systems with the applied control input $u$ and observed output $z$. By virtue of the fact, the prediction error $e\left(t_{i}\right)$ in classical MPE methods is available to specify the criterion functions. In our paper, we consider stochastic control systems, however the prediction error we have is not available because it is defined as 
difference between the output of $\mathfrak{M}(\theta)$ and the optimal (steady-state) estimator of state vector $x$. Hence the object for which we construct a set of $\mathfrak{M}(\theta)$ is not given, i.e. is not available, being the optimal (steady-state) Kalman filter. It is a sort of hidden object to be identified from the observed data $y$. This object, intended to be used in the system feedback, is hidden in the given data source, and we must "extract" it from there.

We do it by constructing an auxiliary error-like process $\varepsilon\left(t_{i}\right)$ such that the relation holds:

$$
J_{\varepsilon}(\theta) \stackrel{\text { def }}{=} \mathrm{E}\left\{\left\|\varepsilon\left(t_{i}\right)\right\|^{2}\right\}=\mathrm{E}\left\{\left\|e\left(t_{i}\right)\right\|^{2}\right\}+\text { Const }
$$

where Const $>0$ denotes a quantity independent of $\mathfrak{M}(\theta)$. Auxiliary Performance Index $J_{\varepsilon}(\theta)$, when minimized instead of Original Performance Index $J_{e}(\theta) \stackrel{\text { def }}{=} \mathrm{E}\left\{\left\|e\left(t_{i}\right)\right\|^{2}\right\}$, theoretically gives the indirect way to estimate the true (i.e., optimal) parameter $\theta^{\circ}$ because the set of $\mathfrak{M}(\theta)$ contains $\mathfrak{M}\left(\theta^{\circ}\right)$ [8], [9].

In this paper, by doing a number of computational experiments, we consider the practicality of this approach as applied to filters that are included into the closed-loop stochastic control systems described by the following equations

$$
\begin{aligned}
x\left(t_{i+1}\right) & =\Phi x\left(t_{i}\right)+\Psi u\left(t_{i}\right)+\Gamma w\left(t_{i}\right) \\
z\left(t_{i}\right) & =H x\left(t_{i}\right)+v\left(t_{i}\right) \\
u\left(t_{i}\right) & =-G_{0} \widetilde{x}\left(t_{i}^{+}\right)
\end{aligned}
$$

where $x\left(t_{i}\right) \in \mathbf{R}^{n}$ is the state vector, $z\left(t_{i}\right) \in \mathbf{R}^{m}$ is the measurement vector, $u\left(t_{i}\right) \in \mathbf{R}^{r}$ is the control input calculated, for lack of optimal filtered estimate $\widehat{x}\left(t_{i}^{+}\right)$and optimal regulator matrix $G_{r}$, through their suboptimal values $\widetilde{x}\left(t_{i}^{+}\right)$ and $G_{0}$, and $\left\{w\left(t_{0}\right), w\left(t_{1}\right), \ldots\right\}$ and $\left\{v\left(t_{1}\right), v\left(t_{2}\right), \ldots\right\}$ are zero-mean independent sequences of independent and identically distributed random vectors $w\left(t_{i}\right) \in \mathbf{R}^{q}$ and $v\left(t_{i}\right) \in \mathbf{R}^{m}$ with covariances $Q$ and $R$ respectively.

\section{General Algorithm}

In this section we present the general computational algorithm for adaptive estimation of parameters that are subject to change. We assume that the uncertainity can reside in matrices $\Phi, \Gamma$ and covariances $Q$ and $R$. The model state estimates under conditions of uncertainty are obtained by the feedback suboptimal filter

$$
\begin{aligned}
\tilde{x}\left(t_{i+1}^{-}\right) & =\Phi_{0} \tilde{x}\left(t_{i}^{+}\right)+\Psi u\left(t_{i}\right) \\
\tilde{x}\left(t_{i}^{+}\right) & =\tilde{x}\left(t_{i}^{-}\right)+K_{0} \nu\left(t_{i}\right) \\
\nu\left(t_{i}\right) & =z\left(t_{i}\right)-H \tilde{x}\left(t_{i}^{-}\right)
\end{aligned}
$$

where control $u\left(t_{i}\right)$ is calculated by a suboptimal control law $u\left(t_{i}\right)=-G_{0} \tilde{x}\left(t_{i}^{+}\right)$. The gain $K_{0}$ is replaced by the result of each iteration (the whole identification process) and $G_{0}$ is recalculated after each iteration according to LQG control theory. The initial values for $K_{0}$ and $G_{0}$ are set as some nominal values which are chosen a'priori to satisfy the stability conditions. The adaptive model is 
appended to this closed-loop system and started with initial state taken from the suboptimal filter (3). It has the following form

$$
\begin{aligned}
\tilde{g}\left(x_{i+1}\right) & =A \hat{g}\left(t_{i}\right)+B u\left(t_{i}\right) \\
\hat{g}\left(t_{i}\right) & =\tilde{g}\left(t_{i}\right)+D \eta\left(t_{i}\right) \\
\eta\left(t_{i}\right) & =z\left(t_{i}\right)-H_{*} \tilde{g}\left(t_{i}\right)
\end{aligned}
$$

where $A=T \Phi T^{-1}, B=T \Psi, H_{*}=H T^{-1}$ and $T$ is the observability matrix defined in [8], [9]. Let us assume that the collective parameter $\theta$ represents the set of adjustable parameters in the model (the Kalman gain $D$ and parameters from matrix $A$ ) indexed accordingly.

Denote the stackable vector of $\eta\left(t_{j}\right), t_{j} \in\left[t_{i-s+1}, t_{i}\right]$ as $\mathrm{H}\left(t_{i-s+1}, t_{i}\right)$ where $s$ is the maximal partial observability index, the model error between the adaptive and suboptimal models then can be written in the following form

$$
\varepsilon\left(t_{i}\right)=\mathcal{N}(D) \mathrm{H}\left(t_{i-s+1}, t_{i}\right)
$$

where $\mathcal{N}(D)$ is the structure transformation of adaptive model gain $D$ as defined in $[8],[9]$.

The sensitivity model that reflects the influence of the adjustable parameters on the model error (5) and in fact is the partial derivatives of vector $\varepsilon\left(t_{i}\right)$ wrt. vector $\theta$, is defined by two types of recursions according to the placement of adjustable parameter. Let $\mu$ denote the sensitivity model state vector, then for parameters $\theta_{j}$ of Kalman gain $D$ we have

$$
\begin{aligned}
\tilde{\mu}_{j}\left(t_{i}\right) & =A \hat{\mu}_{j}\left(t_{i-1}\right) \\
\hat{\mu}_{j}\left(t_{i}\right) & =\left(I-D H_{*}\right) \tilde{\mu}_{j}\left(t_{i}\right)+\frac{\partial D}{\partial \theta_{j}} \eta\left(t_{i}\right)
\end{aligned}
$$

For the adjustable parameters $\theta_{j}$ of transition matrix $A$ the recursions take form

$$
\begin{aligned}
& \tilde{\mu}_{j}\left(t_{i}\right)=\frac{\partial A}{\partial \theta_{j}} \hat{g}\left(t_{i-1}\right)+A \hat{\mu}_{j}\left(t_{i-1}\right) \\
& \hat{\mu}_{j}\left(t_{i}\right)=\left(I-D H_{*}\right) \tilde{\mu}_{j}\left(t_{i}\right)
\end{aligned}
$$

Both recursions start with initial values $\hat{\mu}_{j}\left(t_{0}\right)=0$ for each $\theta_{j}$. Let vector $\xi_{j}\left(t_{i}\right)$ be equal to $-H_{*} \tilde{\mu}_{j}\left(t_{i}\right)$. The history for vectors $\xi_{j}\left(t_{i}\right)$ and $\mathrm{H}\left(t_{i-s+1}, t_{i}\right)$ should be accumulated during the iterations (3)-(7) till $s$ last values are recalculated.

The sensitivity matrix $\mathcal{S}\left(t_{i}\right)$ is computed as follows

$$
\mathcal{S}\left(t_{i}\right)=\left(\frac{\partial \varepsilon\left(t_{i}\right)}{\partial \theta_{j}}\right)=\left(\frac{\partial \mathcal{N}(D)}{\partial \theta_{j}} \mathrm{H}\left(t_{i-s+1}, t_{i}\right)+\mathcal{N}(D) \frac{\partial \mathrm{H}\left(t_{i-s+1}, t_{i}\right)}{\partial \theta_{j}}\right)
$$

where $\frac{\partial \mathrm{H}\left(t_{i-s+1}, t_{i}\right)}{\partial \theta_{j}}$ is the stackable vector $\xi_{j}\left(t_{k}\right), t_{k} \in\left[t_{i-s+1}, t_{i}\right]$.

The gradient model then is defined as the production of the transposed sensitivity matrix $\mathcal{S}\left(t_{i}\right)$ with $\varepsilon\left(t_{i}\right)$

$$
\begin{aligned}
& \mathcal{G}\left(t_{i}\right)=\mathcal{S}^{T}\left(t_{i}\right) \varepsilon\left(t_{i}\right) \\
& \hat{\mathcal{G}}\left(t_{i}\right)=\beta \hat{\mathcal{G}}\left(t_{i-1}\right)+(1-\beta) \mathcal{G}\left(t_{i}\right)
\end{aligned}
$$

where $\beta$ is the exponential smooth factor. 
Three types of adaptation procedures can be used to generate the new estimates for adjustable parameters. Simple stochastic approximation procedure (SSAP)

$$
\pi\left(t_{i}\right)=\hat{\theta}\left(t_{i}\right)-\lambda\left(t_{i+1}\right) \hat{\mathcal{G}}\left(t_{i}\right), \quad \lambda\left(t_{i+1}\right)=1 /\left(t_{i+1}+1\right)
$$

(Here and below $\pi\left(t_{i}\right)$ denotes a trial value for $\hat{\theta}\left(t_{i+1}\right)$ ). The suboptimal adaptation procedure (SAP) is defined for each adjustable parameter $\theta_{j}$ through recursion

$$
\begin{aligned}
p_{j}\left(t_{i+1}\right) & =p_{j}\left(t_{i}\right)+\left\|\frac{\partial \varepsilon\left(t_{i}\right)}{\partial \theta_{j}}\right\|^{2} \\
\pi\left(t_{i}\right) & =\hat{\theta}\left(t_{i}\right)-\operatorname{diag}\left(p_{j}\left(t_{i+1}\right)\right) \hat{\mathcal{G}}\left(t_{i}\right),
\end{aligned}
$$

The optimal adaptation procedure (OAP) is given by

$$
\begin{aligned}
P\left(t_{i+1}\right) & =P\left(t_{i}\right)+\mathcal{S}^{T}\left(t_{i}\right) \mathcal{S}\left(t_{i}\right) \\
P\left(t_{i+1}\right) \Delta \hat{\theta}\left(t_{i}\right) & =-\hat{\mathcal{G}}\left(t_{i}\right) \\
\pi\left(t_{i}\right) & =\hat{\theta}\left(t_{i}\right)+\Delta \hat{\theta}\left(t_{i}\right)
\end{aligned}
$$

The stability condition

$$
\rho\left[\left(I-D H_{*}\right) A\right]<1
$$

for estimate $\pi\left(t_{i}\right)$ should be checked and if new estimates satisfy $(13), \hat{\theta}\left(t_{i+1}\right)=$ $\pi\left(t_{i}\right)$.

\section{$3 \quad$ Numerical Experiments}

We start from the statement of the examples to demonstrate the features of auxiliary performance index (API) algorithm considered in the section 2. The subsection 3.2 is dedicated to the analysis and investigation of the adaptive model estimates behaviour.

\subsection{Numerical Examples}

We consider on the following examples:

E1 Controlled plant. The first order controller model that follows the dynamics of the reference variable and is described by the equations

$$
\begin{aligned}
x\left(t_{i+1}\right) & =\Phi x\left(t_{i}\right)+\Psi u\left(t_{i}\right)+w_{d}\left(t_{i}\right) \\
z\left(t_{i}\right) & =x\left(t_{i}\right)+v_{d}\left(t_{i}\right)
\end{aligned}
$$

where $\Phi=0.82, \Psi=0.18$. Covariances $Q_{d}$ of the zero-mean white Gaussian noise $w_{d}\left(t_{i}\right)$ that is equal to $Q_{d}=0.084 Q(Q$ is the parameter of the experiment) and $R_{d}$ of $v_{d}\left(t_{i}\right)$ are subject to change their values. The reference model which state should be tracked is given by

$$
\begin{aligned}
x_{r}\left(t_{i+1}\right) & =\Phi_{r} x_{r}\left(t_{i}\right)+w_{d r}\left(t_{i}\right) \\
z_{r}\left(t_{i}\right) & =x_{r}\left(t_{i}\right)+v_{d r}\left(t_{i}\right)
\end{aligned}
$$

where $\Phi_{r}=0.61$ and covariances of the noises $Q_{d r}$ and $R_{d r}$ may change their values. Two Kalman gains of controller and reference model should be independently estimated. 
E2 Second order model with possible changes in the covariances of the measurement and state noises is

$$
\begin{aligned}
x\left(t_{i+1}\right) & =\left[\begin{array}{cc}
0 & 1 \\
f_{1} & f_{2}
\end{array}\right] x\left(t_{i}\right)+\left[\begin{array}{l}
0 \\
\beta
\end{array}\right] u\left(t_{i}\right)+\left[\begin{array}{l}
0 \\
\alpha
\end{array}\right] w_{d}\left(t_{i}\right) \\
z\left(t_{i}\right) & =H x\left(t_{i}\right)+v_{d}\left(t_{i}\right)
\end{aligned}
$$

where $\alpha=0.4, \beta=1.0, f_{1}=-0.8$ and $f_{2}=0.1$. The measurement matrix $H$ is $[0,1]$. Kalman gain vector of dimension 2 should be estimated in the adaptation process.

E3 Second order model the same as in E2, with changes in the parameters $f_{1}, f_{2}$ of transition matrix $\Phi$ and covariances of noises. The dimension of the vector $\theta$ of all adjustable parameters is 4 ( $\hat{f}_{1}, \hat{f}_{2}$ and adaptive Kalman gain).

In the examples the optimal stochastic LQG control $u\left(t_{i}\right)$ is calculated from the independent Kalman estimates for the states of corresponding models.

The experiment is started under the initial conditions and then, after the Kalman estimates become stable, switching in the covariances of the noises or the transition matrix (in example E3) occurs. The adaptive estimator is started to identify the new Kalman filter parameters to be used to complete the current iteration step.

\subsection{Properties of Algorithm}

For the analysis of the estimation process behaviour two numerical characteristics are taken into account: the integral percent error (IPE) and the average normalized error (ANE) of parameters estimates. The influence of the signal-noise ratio, dimension of the vector of adjustable parameters, stability conditions on the calculation process characteristics are summarized in this subsection.

Adjustable Parameter Dimension. The influence of the dimension of adjustable parameter that may change its value and should be identified during adaptive process has shown on the graphs Fig. 1 and Fig. 2. On the Fig. 1 the covariance $Q_{d}$ of the state noise is changed from the nominal value of 0.01 to 0.1 , the Fig. 2 represents the case when both covariances $Q_{d}$ and $Q_{d r}$ are changed from 0.01 to 0.1 . The speed and quality of the estimation process is decreased as the number of parameters that change values increases as depicted on the Fig. 1.

Adaptation Procedure. The influence of the adaptation procedure choice on the ANE characteristic of algorithm has shown on the Fig. 3 and the Fig. 4. It can be noted that the selection of OAP in the considered experiments increases the estimation power of the algorithm. The compromise between the number of calculations and the estimation quality is SAP which used in experiments. 


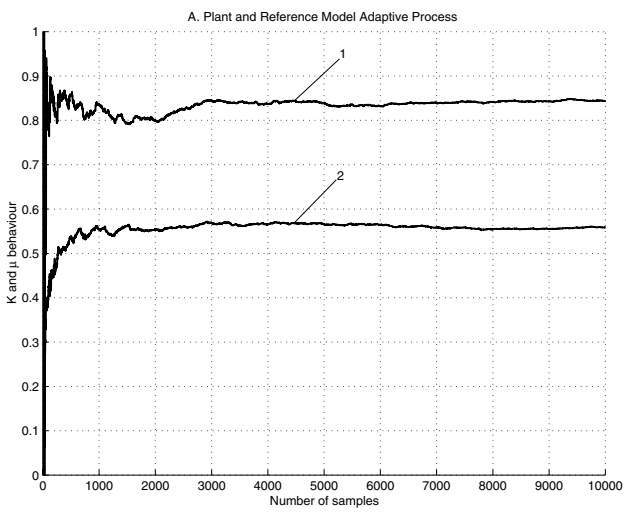

Fig. 1. Example E1. Adaptive estimation process (1-plant model, 2-reference model). The changes in the covariance of plant model.

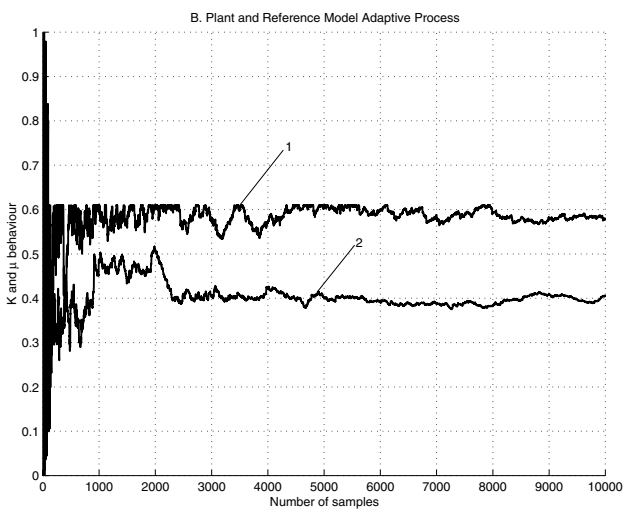

Fig. 2. Example E1. Adaptive estimation process (1-plant model, 2-reference model). The changes in the covariances of plant and reference models.

Signal-Noise Ratio. The IPE characteristic process for different levels of signal-noise ratio for examples E2 and E3 depicted on the Fig. 5 and Fig. 6 shows that the impact on the estimation quality of the algorithm differs in both considered examples. In $A$-graph of Fig. 5 as the $S N R$ increases from 0.01 to 10 , the better performance in the sense of IPE can be observed. When the uncertainty resides also in the transition matrix $\Phi$ the ability of Kalman filter to estimate state of the object becomes crucial. As the noise component in the state equation grows the integral estimation error reaches the unimprovable lower bound (Fig. 6).

Stability Properties. The effect of the unimprovable lower IPE bound is determined also by the stability properties of the object. The contribution of the 


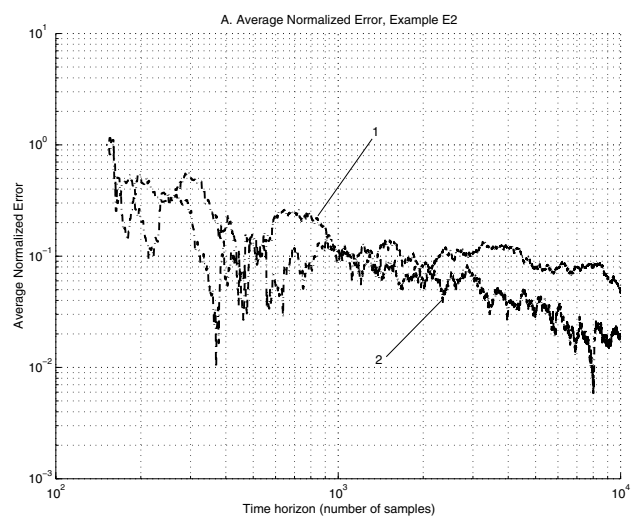

Fig. 3. Example E2. Average normalized error for different adaptation procedures: 1-SAP, suboptimal adaptation procedure, 2-OAP, optimal adaptation procedure, 3-SSAP, simple stochastic adaptation procedure.

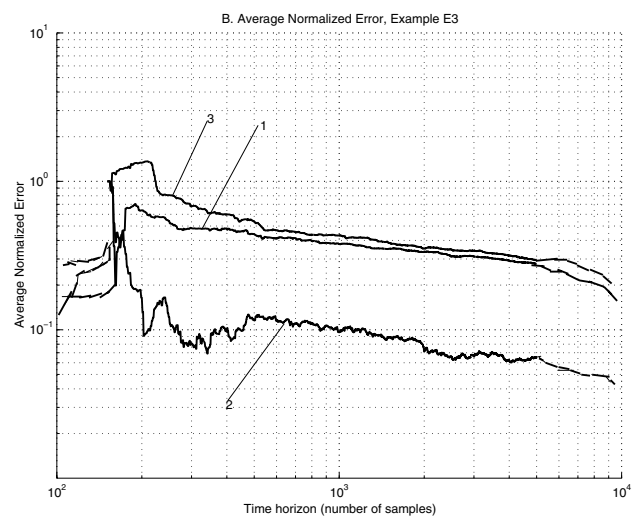

Fig. 4. Example E3. Average normalized error for different adaptation procedures: 1-SAP, suboptimal adaptation procedure, 2-OAP, optimal adaptation procedure, 3-SSAP, simple stochastic adaptation procedure.

dynamic part of the state equation depends on the placement of the object eigenvalues with respect to unit circle on the complex plane. At the fixed level of $S N R$ as the eigenvalues moving to the coordinate origin the quality of obtained estimates decreasing as depicted at the Fig. 7. The influence is more distinct in the case of example E3 again as in the previous subsection.

Initial Values. The results of test runs of the algorithm for different initial values of estimated parameter are shown at Fig. 9 and Fig. 10 at levels SNR 0.1 and 10.0. At the level of 0.1 the algorithm is more sensitive to the initial value than for 10.0 as it is represented at Fig. 10. 


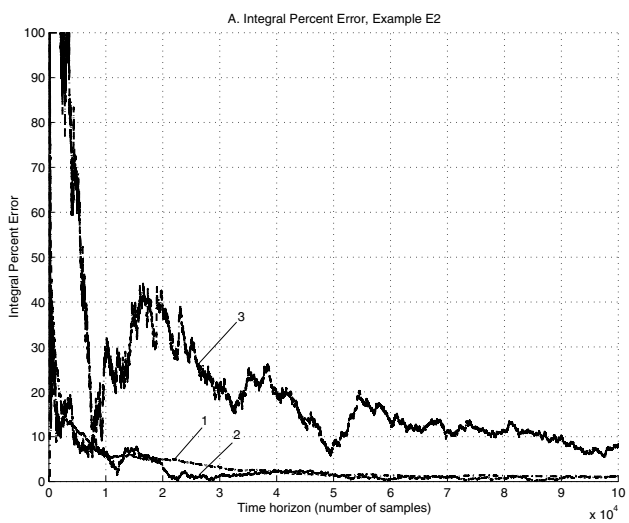

Fig. 5. Example E2. Integral percent error for different level of $S N R: 1-S N R=10,2$ - $S N R=0.1,3-S N R=0.01,4-S N R=0.001$.

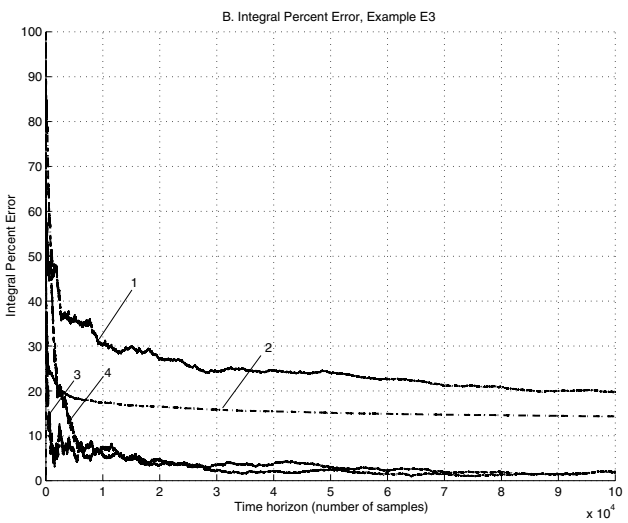

Fig. 6. Example E3. Integral percent error for different level of $S N R: 1-S N R=10,2$ - $S N R=0.1,3-S N R=0.01,4-S N R=0.001$.

\section{Conclusions}

The results reported in the paper show the applicability of the proposed API method to the iterative stochastic control design for the descrete-time closed-loop stochastic control systems. The numerical experiments determined the influence of different factors (the object stability properties, the signal noise ratio, the choice of the initial estimate value) on the algorithm robustness and performance. Some additional experimental effects revealed during the numerical simulation to be theoretically justified. 


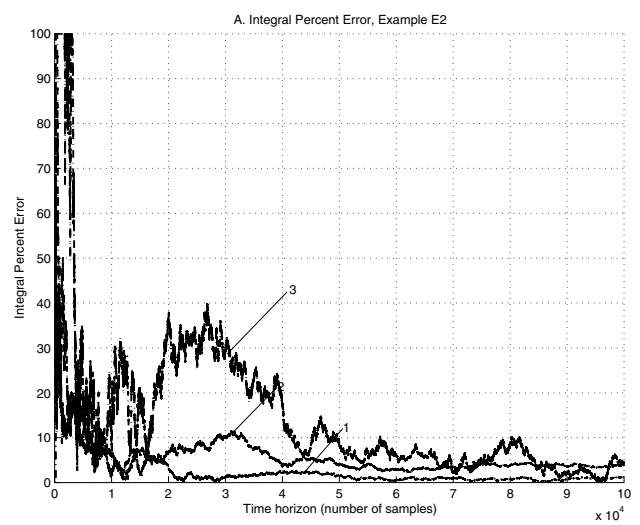

Fig. 7. Example E2. Integral percent error for different level of stability of the object: $1-\rho=0.1,2-\rho=0.2,3-\rho=0.5$.

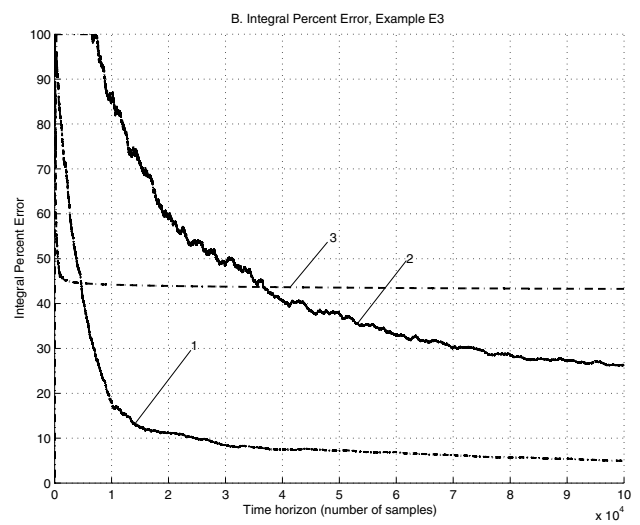

Fig. 8. Example E3. Integral percent error for different level of stability of the object: $1-\rho=0.1,2-\rho=0.2,3-\rho=0.5$.

\section{References}

1. R. R. Schrama. "Accurate identification for control: the necessity of an iterative scheme". IEEE Transactions on Automatic Control, Vol. 37(7), pp. 991-994 (1992).

2. Eykhoff, P. (1974); System Identification; Wiley (pp. 684)

3. Saridis, G.N. (1977); Self-Organizing Control of Stochastic Systems; Marcel Dekker, Inc. (pp. 400)

4. Tsypkin, Ya. Z., E.D. Avedyan and O.V. Gulinsky (1981); On Convergence of the Recursive Identification Algorithms; IEEE Trans. Aut. Control, Vol. AC-26, No. 5 (pp. 1009-1017)

5. Nguyen, V. V. and E.F. Wood (1982); Review and Unification of Linear Identifiability Concepts; SIAM Review, Vol. 24 (pp. 34-51) 


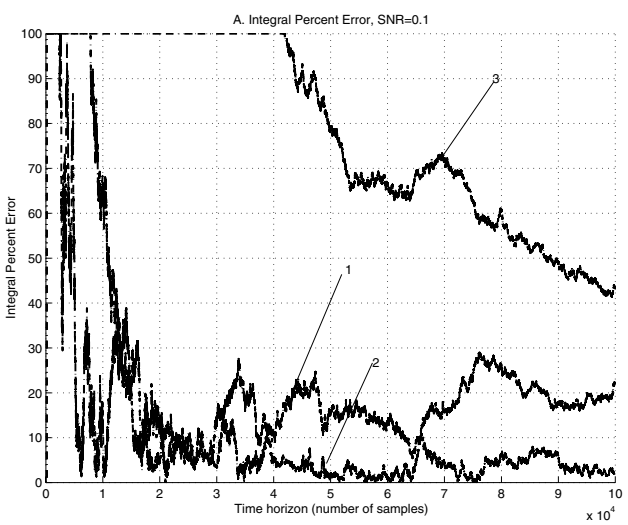

Fig. 9. Example E2. The influence of initial value of estimates for the $S N R=0.1$. 1 $\theta_{0}, 2-5 \theta_{0}, 3-10 \theta_{0}$.

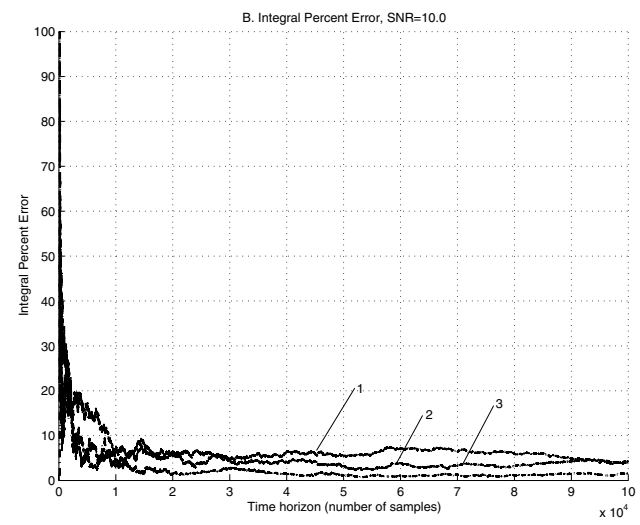

Fig. 10. Example E2. The influence of initial value of estimates for the $S N R=10.0 .1$ - $\theta_{0}, 2-5 \theta_{0}, 3-10 \theta_{0}$.

6. Caines, P.E. (1988); Linear Stochastic Systems; Wiley (pp. 874)

7. Ljung, L. (1978); Convergence Analysis of Parametric Identification Methods; IEEE Trans. Aut. Control, Vol. AC-23, No. 5 (pp. 770-783)

8. Semoushin, I. V. (1987); Active Methods of Adaptation and Fault Detection for Stochastic Control Systems; D.Sc. Dissertation; Leningrad Institute of Aircraft Equipment Engineering (pp. 426) [in Russian]

9. Semoushin, I. V. and J. V. Tsyganova (2000); Indirect Error Control for Adaptive Filtering; Proc. $3^{\text {rd }}$ European Conference on Numerical Mathematics and Advanced Applications, ENUMATH-99, (eds. P. Neittaanmaki, T. Tiihonen and P. Tarvainen), World Scientific, Singapore (pp. 333-340) 